\title{
Resolution, SNR, Signal Averaging and Scan Time in MRI for Metastatic Lesion in Spine: A Case Report in a 74 Years Old Patient
}

\author{
Waseem Zafar ${ }^{1,2 *}$, Ahmed Masood ${ }^{2}$, Basit Iqbal' ${ }^{1}$ and Sohail Murad ${ }^{1}$ \\ 1Department of Nuclear Medicine, Gujranwala Institute of Nuclear Medicine \& \\ Radiotherapy, Pakistan \\ 2Department of Medical Imaging, Medcare International Hospital, Pakistan
}

\section{Case Report}

Volume 3 Issue 1

Received Date: January 29, 2019

Published Date: February 26, 2019

*Corresponding author: Waseem Zafar, Gujranwala Institute of Nuclear Medicine \& Radiotherapy (GINUM), Sialkot Road, Nizampur, Gujranwala, Pakistan, Tel: +92 (300) 4576755; Email: waseem_mt@yaoo.com

\section{Abstract}

Background: MRI image quality is compromise between resolution, scan time, Signal Averaging (NSA/NEX.), SNR and Integrated parallel acquisition technique (SENSE). Any compromise in any of these parameters can lead to poor quality images that can lead to misdiagnosis. The higher the image resolution, the better the small pathologies can be diagnosed, thus it is the goal of imaging a good quality scan by using a standardized protocols.

Methods: The study was conducted in Department of Medical Imaging, Medcare International Hospital, Gujranwala, Pakistan, Gujranwala Institute of Nuclear Medicine \& Radiotherapy (GINUM), Gujranwala, Pakistan.

Case Presentation: We present a case of 74 year old man with severe lower back pain. His initial MRI did not reveal any significant pathology; however a repeat MRI revealed metastatic involvement of the lumbar vertebrae.

Conclusion: Standardized MRI protocols have been developed after much study to optimize all the parameters. Any modification in the protocols to reduce image time is therefore hazardous. It is therefore imperative that they be followed in order to avoid generating suboptimal images.

Keywords: MRI; Signal-to-Noise Ratio; SNR; Resolution; Metastasis; NSA/NEX; IPAT; SENSE Case Report

Abbreviations: MRI: Magnetic Resonance Imaging; STIR: Short-TI Inversion Recovery; SNR: Signal to Noise Ratio; CME: Continuing Medical Education; T1W: T1 Weighted Images; T2W: T2 Weighted Images; NSA: Signal Averaging; SENSE: Sensitivity Encoding; IPAT: Integrated Parallel Acquisition Technique.

\section{Introduction}

Resolution is the ability of human eyes to distinguish one structure from other. In MRI the resolution is determined by the number of voxels in a specified FOV. The higher the image resolution, the better the small pathologies can be diagnosed. Resolution is directly proportional to the number of voxels. Voxel size can be calculated by dividing the FOV by the matrix size (e.g. FOV 320 , Matrix $320 \times 320$, voxel size is $320 / 320=1 \mathrm{~mm}$ ). There are two resolution parameters used in MRI for the production of a 2D image i.e. basic resolution \& phase resolution $[1,2]$. 


\section{Clinical Radiology \& Imaging Journal}

\section{Basic Resolution}

Basic resolution is the number of voxels in redout direction. Basic resolution determines the size of the image matrix. Basic resolution is inversely proportional to the size of the voxel [2].

\section{SNR \& Basic Resolution}

SNR is inversely proportional to the basic resolution. In other words SNR is directly proportional to the voxel size, increasing the base resolution will reduce the voxel size therefore the SNR of the image $[3,4]$ will be reduced (Figure 1).
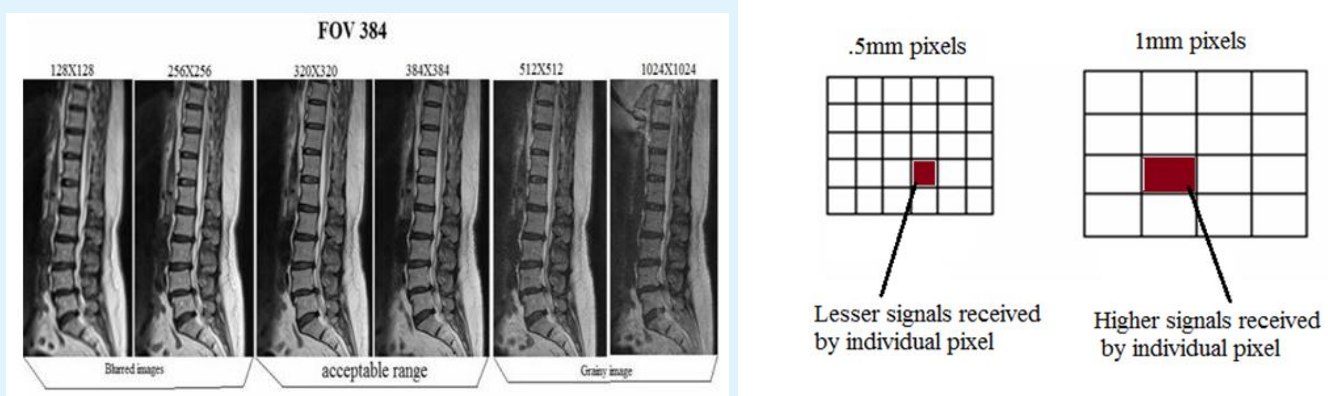

Figure 1: A comparison of the effect of various matrix sizes on image quality when keeping a constant FOV.

If we compare a $256 \times 256$ and $128 \times 128$ matrix formats, the voxel size of $128(2 \times 2 \mathrm{~mm})$ matrix is four times larger than the $256(1 \times 1 \mathrm{~mm})$ matrix but there are only half phase encoding steps. Therefore the SNR is calculated by $4 \sqrt{2}=2.82$ (Table 1 ).

\begin{tabular}{|c|c|}
\hline Matrix & Relative SNR \\
\hline $128 \times 128$ & $2.82(1 \times 2.8=2.82)$ \\
\hline $256 \times 256$ & 1 \\
\hline $512 \times 512$ & $0.35(1 / 2.82=3.5)$ \\
\hline
\end{tabular}

Increasing the basic resolution will increase the image quality. Increasing the resolution more than the acceptable range will produce noisy or grainy image due to low SNR and reducing the basic resolution more than the acceptable range will produce a blurry image due to high SNR [4]. Increasing the basic resolution will result in a prolonged scan time. Ways to improve a low resolution / blurred image. Improvement in scan time leads to a higher resolution and sharper images (Figure 2).

Table 1: Relationship between Matrix size and SNR

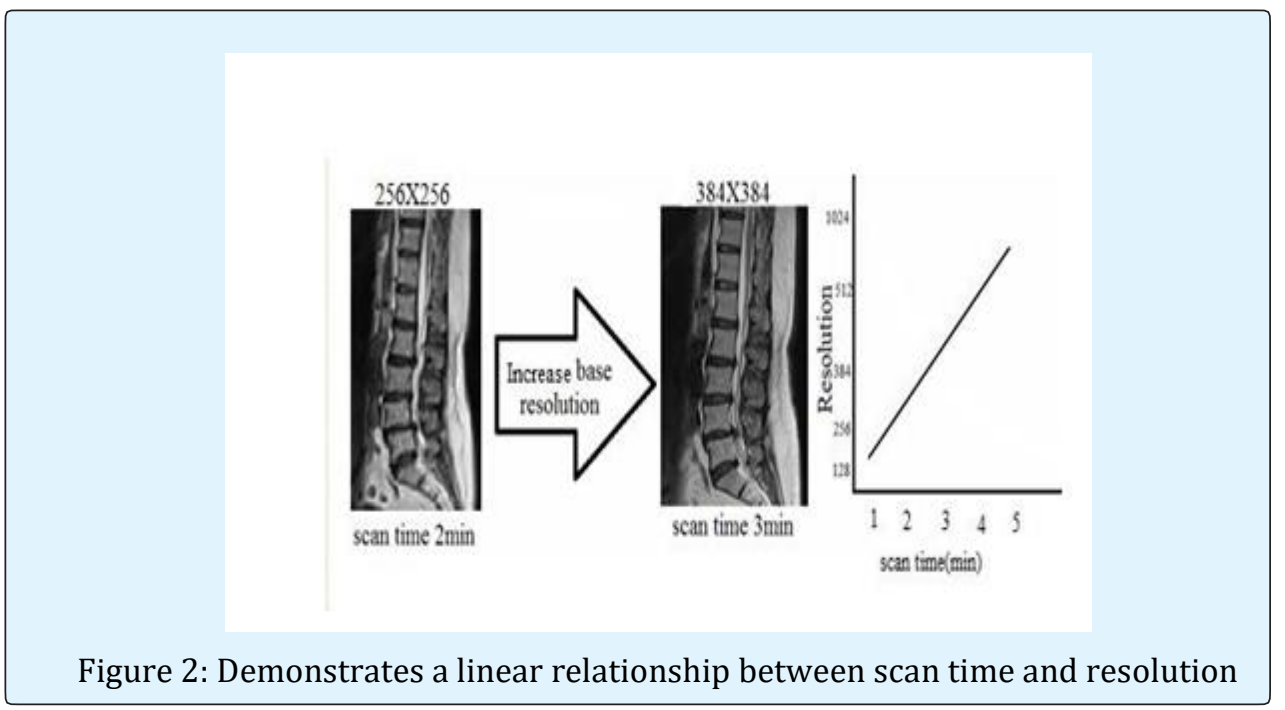

Decreasing FOV reduces the voxel size and SNR therefore the image will become sharper (Figure 3). 


\section{Clinical Radiology \& Imaging Journal}
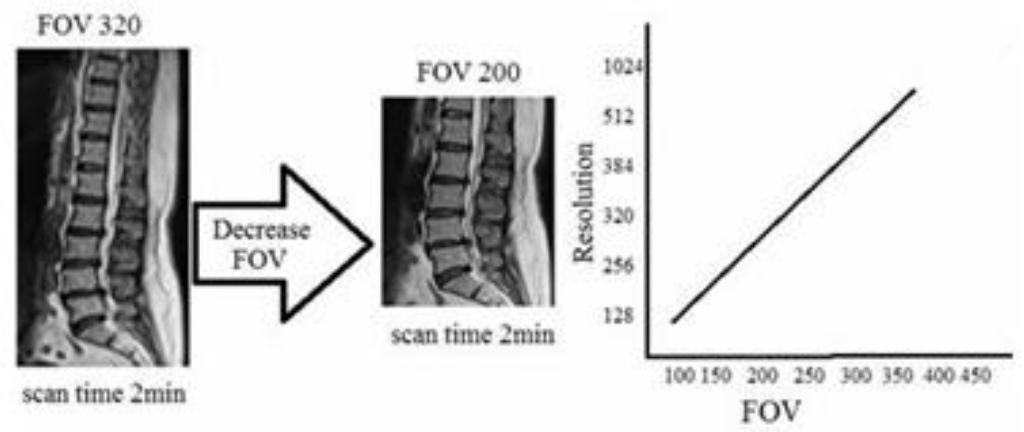

Figure 3: Demonstrates a relationship between FOV and resolution.

\section{SNR \& Phase Resolution}

Decreasing the phase resolution will reduce the image quality and scan time. Reducing the phase resolution will increase the voxel size therefore the SNR will increase considerably (Figures $4 \& 5$, Table 2).

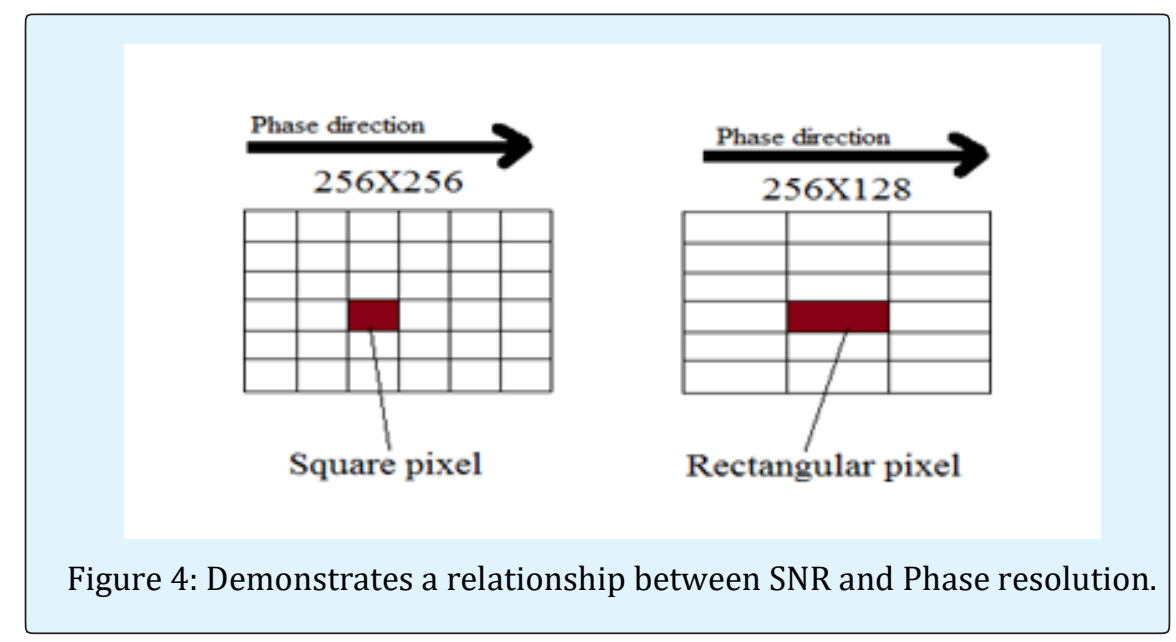

\begin{tabular}{|c|c|c|c|c|}
\hline FOV readout & FOV phase & Phase resolution & Matrix Size & Pixel (mm) \\
\hline $256 \mathrm{~mm}$ & $100 \%$ & $100 \%$ & $256 \times 256$ & $1 \times 1$ \\
\hline $256 \mathrm{~mm}$ & $100 \%$ & $75 \%$ & $256 \times 192$ & $1 \times 1.33$ \\
\hline $256 \mathrm{~mm}$ & $100 \%$ & $50 \%$ & $256 \times 128$ & $1 \times 2$ \\
\hline $256 \mathrm{~mm}$ & $100 \%$ & $25 \%$ & $256 \times 64$ & $1 \times 4$ \\
\hline
\end{tabular}

Table 2: Demonstrates a relationship between SNR and Phase resolution. 


\section{Clinical Radiology \& Imaging Journal}

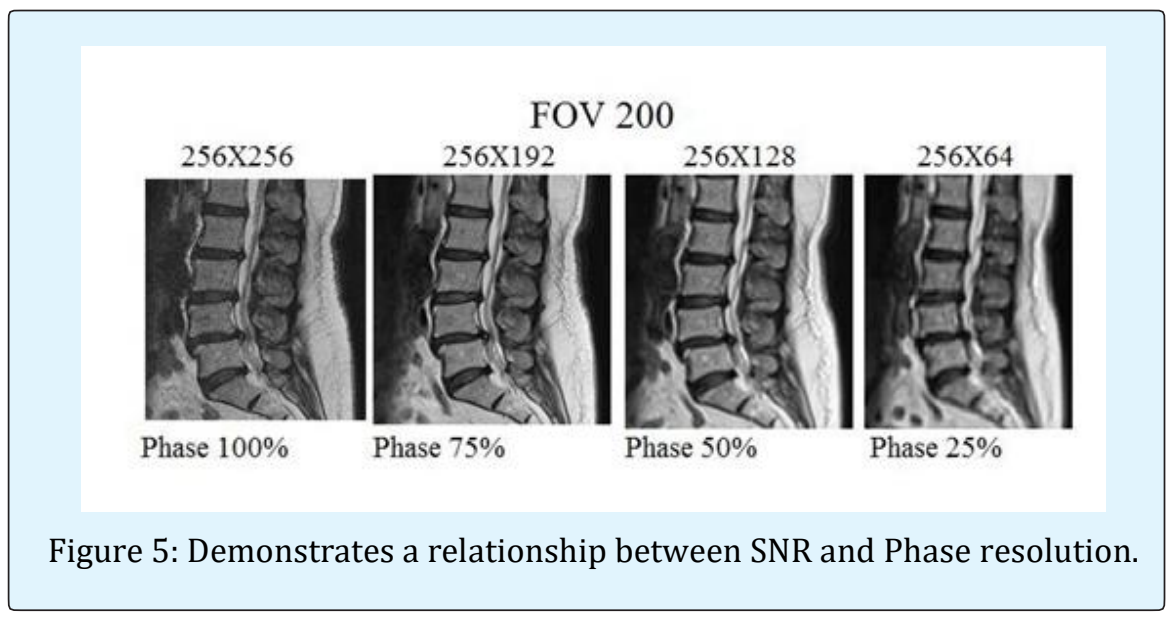

\section{Signal Averaging}

A signal to noise improvement method that is accomplished by taking the average of several FID`s made under similar conditions to suppress the effects of random variations or random artifacts. It is a common method to increase the SNR by averaging several measurements of the signal. The number of averages is also referred to as the number of excitations (NEX) or the number of acquisitions (NSA). Doubling the number of acquisitions will increase the SNR by the $\sqrt{2}$. The approximate amount of improvement in signal to noise (SNR) ratio is calculated as the square root of the number of excitations [5].

By using multiple averages, respiratory motion can be reduced in the same way that multiple averages increase the signal to noise ratio. NEX/NSA will increase SNR but will not affect contrast unless the tissues are being lost in noise (low CNR). Scan time scales directly with NEX/NSA and SNR as the square root of NEX/NSA. The use of phase array coils allows the number of signal averages to be decreased with their superior SNR and resolution, thereby decreasing.

\section{Sense (IPAT)}

With SENSE, two or more coil elements acquire imaging data simultaneously, enabling a scan time reduction by a factor of up to the number of coil elements used. The major negative point of parallel imaging techniques is that they diminish SNR in proportion to the numbers of reduction factors [4]. $\mathrm{R}$ is the factor by which the number of k- space samples is reduced. In standard Fourier imaging reducing the sampling density results in the reduction of the FOV, causing aliasing. As long as the actual phase percentage is close to the requested phase percentage, the resulting image will be fine. The difference can increase when higher TFE-factors are used, and in that case you might need to tune the sequence (change number of phases, spatial resolution and/or SENSE factor) to avoid that the actual phase percentage is much lower than the requested phase percentage: this could lead to increased temporal blurring.

\section{Parameters Benefits and Trade-offs}

The relationships between MR imaging parameters are complex. The table shows the effects of increasing or enabling a parameter (set to "Yes") on scan time, resolution, signal-to-noise ratio (SNR) and artifact level for some parameters (Table 3).

\begin{tabular}{|c|c|c|c|c|}
\hline \multicolumn{2}{|c|}{ \lower, $\uparrow$ higher, = unchanged, • more information available in following Table } \\
\hline Parameter & Scan time & Resolution & SNR & Artifacts \\
\hline NSA & $\uparrow$ & $=$ & $\uparrow$ & $\downarrow$ \\
\hline REST & $\uparrow$ & $=$ & $=$ & $\downarrow$ \\
\hline Voxel Size & $\downarrow$ & $\downarrow$ & $\uparrow$ & $\uparrow / \downarrow$ \\
\hline FOV (In combination with fixed matrix size)* & $=$ & $\downarrow$ & $\uparrow$ & $=$ \\
\hline FOV (In combination with fixed voxel size) & $\uparrow$ & $=$ & $\uparrow$ & $=$ \\
\hline Rectangular FOV (\%) & $\uparrow$ & $=$ & $\uparrow$ & $=$ \\
\hline
\end{tabular}




\section{Clinical Radiology \& Imaging Journal}

\begin{tabular}{|c|c|c|c|c|}
\hline Parameter & Scan time & Resolution & SNR & Artifacts \\
\hline Scan Percentage (\%) & $\uparrow$ & $\uparrow$ & $\downarrow$ & $=$ \\
\hline Slice thickness & $=$ & $\downarrow$ & $\uparrow$ & $=$ \\
\hline Scan Matrix & $\uparrow$ & $\uparrow$ & $\downarrow$ & $=$ \\
\hline Halfscan & $\downarrow$ & $=$ & $\downarrow$ & $\uparrow$ \\
\hline SMART & $=$ & $=$ & $\uparrow$ & $\downarrow$ \\
\hline Water Fat Shift & $=$ & $=$ & $\uparrow$ & $=$ \\
\hline 3D Slices & $\uparrow$ & $=$ & $\downarrow$ & $=$ \\
\hline SE Flip Angle & $=$ & $=$ & $\downarrow$ & $\downarrow$ \\
\hline Flowcomp FFE, TSE & $=$ & $=$ & $=$ & $\downarrow$ \\
\hline Flowcomp SE & $=$ & $=$ & $\uparrow / \downarrow$ & $\uparrow / \downarrow$ \\
\hline Partial Echo & $=$ & $=$ & $=$ & $\downarrow$ \\
\hline SPIR/ ProSet & $\uparrow$ & $=$ & $\downarrow$ & $=$ \\
\hline SENSE & $\downarrow$ & $=$ & & $\downarrow$ \\
\hline
\end{tabular}

The effect of parameter FOV can differ depending on the way of working: you can either adjust the Matrix size or the voxel size. The table illustrates the effects.

\begin{tabular}{|c|c|c|c|c|c|c|c|}
\hline Way of working & FOV & Scan matrix & Pixel size & TE & TR & Scan time & SNR \\
\hline Matrix size & $\downarrow$ & $=$ & $\downarrow$ & $\uparrow 1)$ & $\uparrow 2)$ & $\uparrow$ & $\downarrow 3)$ \\
\hline Voxel size & $\downarrow$ & $\downarrow$ & $=$ & $=$ & $=$ & $\downarrow$ & $\downarrow 4)$ \\
\hline
\end{tabular}

Footnotes: 1) This is valid in case of TE= shortest.

For TE= user defined, this could lead to a conflict.

2) This is valid in case of TR= Shortest.

3) This is caused by smaller voxels.

4) This is caused by the reduction of scan time.

Table 3: Parameters benefits and trade-offs.

\section{Instrumentation}

\section{MRI Systems}

- Achieva (Philips) $1.5 \mathrm{~T}$

- Magnetom-C (SIEMENS) 0.35T

\section{Objective}

The study is designed with following objectives:

a) To detect the malignant lesions in spine by using standard protocols to obtain good quality images?

b) How system optimization can influence the delectability, sensitivity and reliability of an imaging system of MRI?

\section{Methods}

The study was conducted in Department of Medical Imaging, Medcare International Hospital, Gujranwala, Pakistan, Gujranwala Institute of Nuclear Medicine \& Radiotherapy (GINUM), Gujranwala, Pakistan.

\section{Case Presentation}

We present the case of a 74-year-old male with a history of severe lower back pain, bony aches, vomiting and abdominal pain. He was referred for an MRI spine from a local hospital to evaluate a suspicious lytic lesion seen on x-ray. Two independent observers observed the both studies separately and gave their comments regarding the MRI studies. A first or previous MRI spine done two weeks earlier (using an Achieva Philips 1.5 Tesla system) had failed to characterize the lesion adequately by the first observer, simply showing intervertebral disc bulges (Figures $6 \& 7$ ).

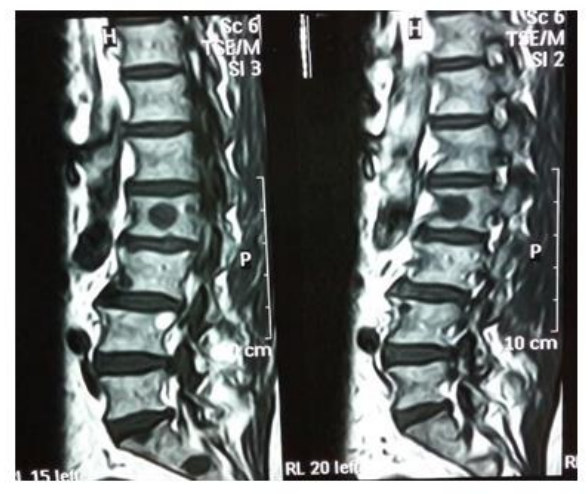

Figure 6: T1 weighted sagittal images performed with the 1.5 Tesla Achieva (Philips) machines, which showing only disc bulges and single lesion at L2V, but not clear. 


\section{Clinical Radiology \& Imaging Journal}

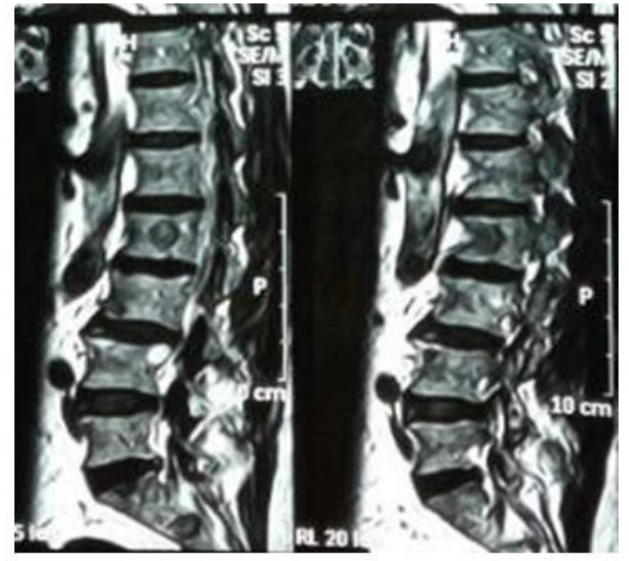

Figure 7: T2 weighted sagittal images performed with the 1.5 Tesla machine which showing only disc bulges and single lesion at $\mathrm{L} 2 \mathrm{~V}$, but not clear.

A repeat MRI spine was performed at our institution using a 0.35 Tesla (Magnetom-C (SIEMENS) machine was using standardized protocol. The second scan revealed by second observer metastatic lesions in multiple dorsolumbar vertebrae (Figures 8-12). Subsequently the earlier MRI was reviewed which also seemed to have similar changes but far less clear. The same was communicated to the referring physician who ordered a CT chest, abdomen \& pelvis including biphasic to diagnose the site of primary lesion, which turned out to be cancer of head pancreas. Bony metastatic lesions were subsequently confirmed in bone scan performed at a local nuclear medicine facility by author BI.

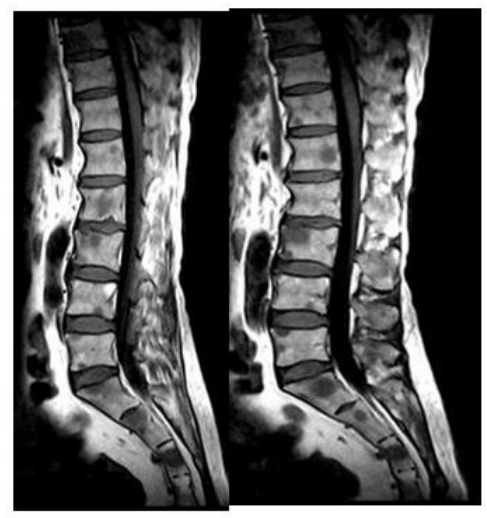

Figure 8: T1 weighted sagittal images performed with 0.35 Tesla Magnetom-C (SIEMENS) machine which showing metastatic lesion at multiple levels of lumbosacral spine.

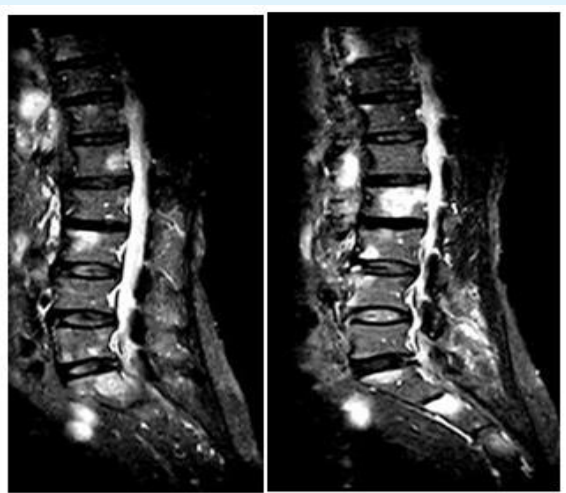

Figure 9: (T2 weighted and STIR Sagittal images performed with the 0.35 Tesla Magnetom-C (SIEMENS) MRI machine which showing hyper intense signals are metastatic lesions at multiple levels of vertebrae of lumbosacral spine.
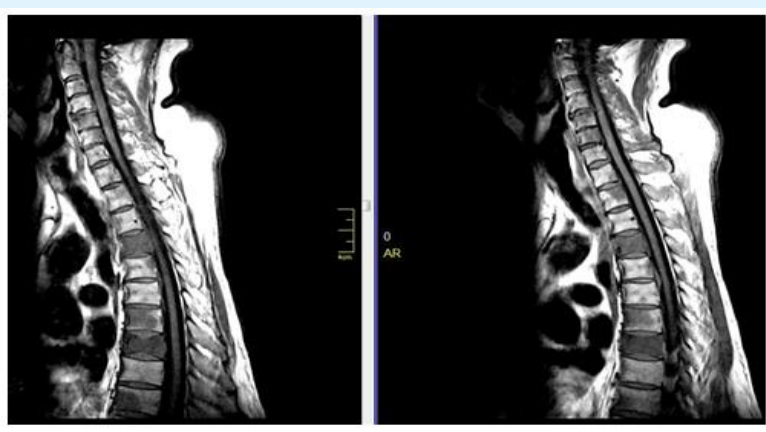

Figure 10: T1 weighted sagittal images of dorsal spine performed with the 0.35 Tesla Magnetom-C (SIEMENS) machine. Which showing metastatic lesion at multiple levels of dorsal spine.
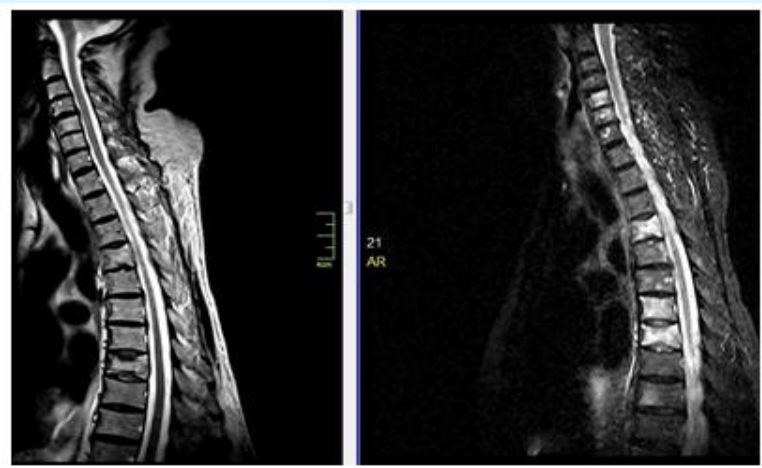

Figure 11: T2 weighted (Left) \& T2 STIR weighted sagittal images of dorsal spine performed with the 0.35 Tesla Magnetom-C (SIEMENS) machine, which showing metastatic lesion at multiple levels of dorsal spine. 


\section{Clinical Radiology \& Imaging Journal}
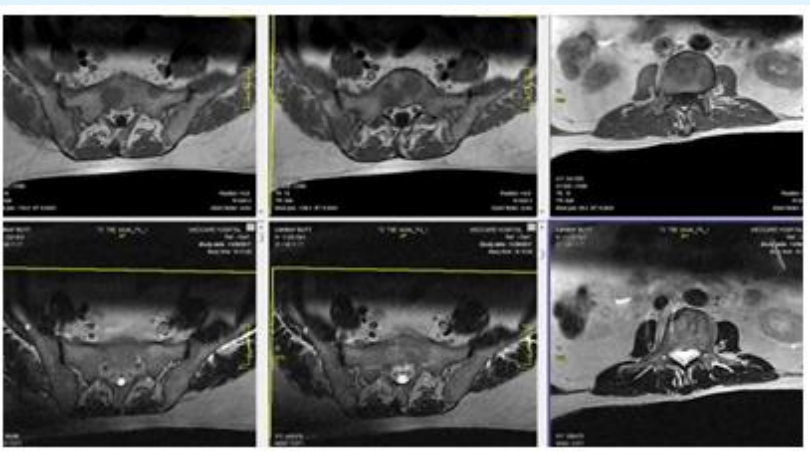

Figure 12: T1weighted (upper row) and T2 weighted (lower row) axial images of spine performed with the the 0.35 Tesla Magnetom-C (SIEMENS) machine, which showing metastatic lesion.
Both the MRI studies were technically analyzed by author WZ to discover the cause of missing metastatic lesions in the first MRI scan. It was observed that the image quality was suboptimal due to a compromise to minimize resolution, SNR, signal averages (NSA, NEX) and also applying SENSE technique to shorten the scan time, due to which there were blurry and poor quality images were acquired and information were lost which lead to misdiagnosis (Tables 5 \& 6).

Following were the parameters of $\mathrm{T} 1 \mathrm{w}$ sequence used of both MRI modalities

\begin{tabular}{|c|c|c|c|c|c|c|c|c|c|c|c|c|c|c|c|}
\hline $\begin{array}{l}\text { Sr. } \\
\text { No }\end{array}$ & $\begin{array}{c}\text { MRI } \\
\text { Systems }\end{array}$ & Tesla & $T R$ & TE & $\begin{array}{l}\text { Scan } \\
\text { Time }\end{array}$ & $\begin{array}{c}\text { NSA/N } \\
\text { EX } \\
\text { (Avg) }\end{array}$ & \begin{tabular}{|c|}
$\begin{array}{c}\text { Matrix } \\
\text { (Base } \\
\text { Resolution) }\end{array}$ \\
\end{tabular} & $\begin{array}{c}\text { Phase } \\
\text { Resolutio } \\
n\end{array}$ & Filter & RFOV & $\begin{array}{l}\text { Phase } \\
\text { FOV }\end{array}$ & $\begin{array}{l}\text { VOXE } \\
\text { L Size }\end{array}$ & $\begin{array}{c}\text { Slice } \\
\text { Thickness }\end{array}$ & SENSE & COIL \\
\hline 1 & $\begin{array}{l}\text { Achieva } \\
\text { (Philips) }\end{array}$ & $1.5 \mathrm{~T}$ & 400 & 11 & $\begin{array}{l}1.10 \\
\min \end{array}$ & 2 & 256 & 70 & $\begin{array}{l}\text { Smootheni } \\
\text { ng applied }\end{array}$ & 309 & $\begin{array}{c}311 / 1 \\
1\end{array}$ & $\begin{array}{c}0.6 \\
\mathrm{~mm}\end{array}$ & 4 & $\begin{array}{l}\text { SENSE- } \\
\text { Spine }\end{array}$ & $\begin{array}{c}\text { Surface coil } \\
\text { for spine }\end{array}$ \\
\hline 2 & $\begin{array}{l}\text { Magnetom- } \\
\text { C(SIEMENS) }\end{array}$ & $0.35 \mathrm{~T}$ & 444 & 18 & $\begin{array}{c}4: 18 \\
\min \end{array}$ & 3 & 320 & 90 & $\begin{array}{l}\text { Medium } \\
\text { Smootheni } \\
\text { ng applied }\end{array}$ & 350 & 100 & $\begin{array}{l}0.53 \\
\mathrm{~mm}\end{array}$ & 4.5 & $\mathrm{~N} / \mathrm{A}$ & $\begin{array}{c}\text { Phased } \\
\text { array } \\
\text { surface }+ \\
\text { body coil }\end{array}$ \\
\hline
\end{tabular}

Table 5: Comparison of Parameters of T2w sequence of both modalities.

Following were the Parameters of T2w sequence used for both MRI modalities

\begin{tabular}{|c|c|c|c|c|c|c|c|c|c|c|c|c|c|c|c|}
\hline $\begin{array}{l}\text { Sr. } \\
\text { No }\end{array}$ & $\begin{array}{c}\text { MRI } \\
\text { Systems }\end{array}$ & Tesla & TR & $\mathrm{TE}$ & Scan & \begin{tabular}{|c|} 
NSA/ \\
NEX \\
(Avg) \\
\end{tabular} & $\begin{array}{c}\text { Matrix } \\
\text { (Base } \\
\text { Resolution }\end{array}$ & $\begin{array}{c}\text { Phase } \\
\text { Resolution }\end{array}$ & Filter & RFOV & $\begin{array}{c}\text { Phase } \\
\text { Fov }\end{array}$ & $\begin{array}{c}\text { VOXEL } \\
\text { Size }\end{array}$ & $\begin{array}{c}\text { Slice } \\
\text { Thickness }\end{array}$ & SENSE & COIL \\
\hline 1 & $\begin{array}{l}\text { Achieva } \\
\text { (Philips) }\end{array}$ & $1.5 \mathrm{~T}$ & 2210 & 120 & $\left\{\begin{array}{l}1.50 \\
\min \end{array}\right.$ & 3 & 256 & 70 & $\begin{array}{c}\text { moothe } \\
\text { ing } \\
\text { applied }\end{array}$ & 309 & $311 / 11$ & $0.6 \mathrm{~mm}$ & 4 & $\begin{array}{l}\text { SENSE- } \\
\text { Spine }\end{array}$ & $\begin{array}{c}\text { Surface coil } \\
\text { for spine }\end{array}$ \\
\hline 2 & $\begin{array}{l}\text { Magnetom- } \\
\text { (SIEMENS }\end{array}$ & b.35T & 3350 & 13 & $\begin{array}{l}6: 30 \\
\min \end{array}$ & 5 & 320 & 90 & & 350 & 100 & $0.53 \mathrm{~mm}$ & 4.5 & $\mathrm{~N} / \mathrm{A}$ & $\begin{array}{c}\text { Phased array } \\
\text { surface }+ \\
\text { body coil }\end{array}$ \\
\hline
\end{tabular}

Table 6: Comparison of Parameters of T2w sequence of both modalities.

\section{Discussion}

a. Observations by comparing the parameters (Tables 5 \& 6) and images (Figures 6-12) of both studies demonstrate that the operator of 1.5T MRI System Achieva (Philips) shorten the scan time by decreasing signal averages (NSA or NEX), matrix size, phase resolution, increasing voxel size and by using IPAT or SENSE technique which leads to poor quality images. b. By reducing number of averages (NSA or NEX) decrease the signal to noise ratio and increasing the respiratory motion artifact which leads to blurry images in 1.5T MRI System Achieva (Philips) (Tables $3,5 \& 6)$.

c. By reduction the matrix size from 320 to 256 \& phase resolution to decrease the scan time and also diminish 


\section{Clinical Radiology \& Imaging Journal}

the resolution by increasing the voxel size (Tables 3, 5 \& 6).

d. In comparison to $0.35 \mathrm{~T}$ Magnetom-C (SIEMENS) scanners, a SENSE (IPAT) technique) has applied to shorten the scan time which also diminished SNR in1.5T MRI System Achieva (Philips) (Tables 5 \& 6).

\section{Conclusion}

This case illustrates the need for using standard protocols with optimal timing of sequence to obtain good quality images with high resolution within acceptable range of SNR for proper diagnosis of small pathologies and metastatic lesions of spine. Many MRI technologists shorten the scan time by decreasing resolution, SNR, signal averages (NSA or NEX) and by using the SENSE (IPAT) technique which leads to blurry or poor resolution images were acquired and information were lost which lead to misdiagnosis. Continuous CME for technologists is needed to keep them abreast of the latest guidelines and techniques to aware of MRI parameters benefits and trade-offs.

\section{Conflicts of Interest}

The authors have no conflicts of interest to disclose.
Informed consent was obtained from the patient to publish this case in a medical journal.

\section{Ethical Approval}

Ethical approval is not required at our institution for publishing a case report in a medical journal.

\section{References}

1. IAEA (2014) Diagnostic Radiology Physics. International Atomic Energy Agency, Vienna.

2. Hashemi RH, WG Bradley, CJ Lisanti (2010) MRI: The Basics. Lippincott Williams \& Wilkins, Philadelphia.

3. McRobbie DW, Moore EA, Graves MJ, Prince MR (2006) MRI from Picture to Proton $2^{\text {nd }}$ (Edn), Cambridge University Press, New York.

4. Li BS, Regal J, Gonen O (2001) SNR versus resolution in 3D $1 \mathrm{H}$ MRS of the human brain at high magnetic fields. Magn Reson Med 46(6): 1049-1053.

5. Murphy K, Bodurka J, Bandettini PA (2007) How long to scan? The relationship between fMRI temporal signal to noise and necessary scan duration. Neuro Image 34(2): 565-574.

\section{Consent for Publication}

